\title{
Evaluación de daños y actuaciones de rehabilitación en la iglesia de San Nicolás de Eduardo Torroja (Gandía, 1962)
}

\author{
Damage assessment and restoration actions in the church of San Nicolas \\ by Eduardo Torroja (Gandia, 1962)
}

\author{
$\underline{\text { B. Serrano-Lanzarote }}^{(*)}$, E. Fenollosa Forner ${ }^{(*)}$, F. Arnau Paltor ${ }^{(*)}$
}

RESUMEN

La iglesia de San Nicolás de Gandía (1962), es la obra póstuma del ingeniero Eduardo Torroja Miret, en colaboración con el arquitecto Gonzalo Echegaray Comba. El templo se caracteriza por su singular estructura: dos láminas plegadas de hormigón armado postesado, apoyadas en los testeros, salvando una luz de 29 m. En el año 1996, la detección de fisuras en el hormigón y desprendimientos de recubrimientos de las armaduras, hicieron aconsejable una inspección y evaluación exhaustiva de las lesiones que presentaba el edificio, mayormente producidas por la corrosión de las armaduras del hormigón, y que concluyó recomendando la reparación de las mismas.

El presente trabajo explica el sistema estructural del templo, describe los daños detectados en la estructura, expone el proyecto de rehabilitación que se elaboró y muestra las obras que se ejecutaron durante la intervención. Las obras finalizaron justo para la celebración del 50 aniversario de su construcción en 2012.

Palabras clave: Láminas plegadas; hormigón postesado; corrosión; rehabilitación; conservación; Movimiento Moderno.

\section{ABSTRACT}

The church of San Nicolas in Gandia (1962), is the posthumous work of Eduardo Torroja Miret, engineer, in collaboration with Gonzalo Echegaray Comba, architect. The temple is characterized by its unique structure: two folded sheets of post-tensioned concrete, supported on the side walls, with 29 meters span. In 1996, the detection of cracks in the concrete and spalling, made it advisable an inspection and evaluation of the damages detected in the building, mostly caused by the corrosion of reinforcement concrete, and concluded by recommending repair them.

This paper explains the structural system of the temple, describes the identified damages in the structure, exposes the refurbishment project and displays the works executed during the intervention. The work was completed just in time for the 5oth anniversary of its construction in 2012.

Keywords: Folded-plates; prestressed-concrete; corrosion; refurbishment; conservation; Modern Movement.

${ }^{(*)}$ Universidad Politécnica de Valencia (España).

Persona de contacto/Corresponding author: apserlan@mes.upv.es (B. Serrano-Lanzarote)

Cómo citar este artículo/Citation: Serrano-Lanzarote, B., Fenellosa Forner, E., Arnau Paltor, F. (2016). Evaluación de daños y actuaciones de rehabilitación en la iglesia de San Nicolás de Eduardo Torroja (Gandía, 1962). Informes de la Construcción, 68(541): e130, doi: http://dx.doi.org/10.3989/ic.14.139.

Licencia / License: Salvo indicación contraria, todos los contenidos de la edición electrónica de Informes de la Construcción se distribuyen bajo una licencia de uso y distribución Creative Commons Reconocimiento no Comercial 3.o. España (cc-by-nc). 


\section{INTRODUCCIÓN}

En el presente artículo se expondrán los daños que presentaba la estructura de la iglesia de San Nicolás del Grau de Gandía, detectados en 1996, y el proyecto de rehabilitación integral llevado a cabo entre los años 2002 y 2006.

La Iglesia de San Nicolás es de especial interés por ser la última obra del ingeniero Eduardo Torroja Miret (1) y por su singular tipología estructural, a base de láminas plegadas de hormigón. La intervención del arquitecto Gonzalo Echegaray Comba proporcionó al proyecto una gran calidad arquitectónica. Constituye un interesante ejemplo de arquitectura del Movimiento Moderno, puesto en valor gracias a la labor de investigación de la catedrática Carmen Jordá y la consecuente inclusión de la obra en el Registro DOCOMOMO Ibérico relativo a los Equipamientos (2) (3) (4).

La Iglesia fue construida entre 1959 y 1962. Está situada en la desembocadura del Río San Nicolás en el Grau de Gandía, en un ambiente marino, caracterizado por elevados porcentajes de humedad y de contenidos de cloruros, cuyos síntomas se manifestaban en la corrosión generalizada que presentaban las armaduras de la estructura. Durante sus primeros 35 años, la Iglesia no fue sometida a labores de mantenimiento de importancia. Los daños observados en 1996 motivaron una evaluación exhaustiva del estado del edificio. El trabajo fue encargado a los Laboratorios Intemac.

El informe resultante (5) sirvió como base para el proyecto de rehabilitación integral de la iglesia, obra del arquitecto Ignacio Lafuente Niño (6) (7) (8). El proyecto no sólo pretendía devolver a la iglesia su imagen original, mediante la reparación de los daños y acabados, sino también la modernización de instalaciones y la eliminación de los elementos impropios que distorsionaban la imagen de este conjunto de indudable valor arquitectónico. El proceso de reparación, al que fue sometida dicha estructura, consistió fundamentalmente en la eliminación del hormigón deteriorado hasta alcanzar la posición de las armaduras, que fueron limpiadas del óxido, para proceder finalmente a su reconstrucción y protección frente al desarrollo de futuros procesos de corrosión. Las obras se llevaron a cabo entre 2002 y 2006 de forma que se pudo celebrar el 50 aniversario de su construcción con una imagen completamente renovada (9).

La documentación necesaria para la realización de este estudio se ha obtenido de los archivos del Instituto de Ciencias de la Construcción Eduardo Torroja y del Archivo Histórico del CEHOPU del CEDEX, ambos en Madrid. Así mismo se ha consultado el Archivo Histórico de Gandía, el Archivo de la parroquia de San Nicolás y la documentación personal del arquitecto autor del proyecto de rehabilitación del edificio.

\section{TORROJA Y LA IGLESIA DE SAN NICOLÁS}

Eduardo Torroja Miret (1899-1961), ingeniero de caminos, gozó de un gran prestigio internacional. Compaginó el ejercicio de la profesión junto con la docencia en la Escuela de Caminos de Madrid y la investigación en el campo del hormigón armado, en el Instituto Técnico de la Construcción y la Edificación, del que fue fundador y primer director (actual- mente Instituto de Ciencias de la Construcción Eduardo Torroja, IETcc). Fue, con Franz Dischinger, uno de los pioneros de las estructuras laminares de pared delgada (10) y junto a Félix Candela, Anton Tedesco y Pier Luigi Nervi, el autor de las más elegantes. Entre las más conocidas están la cubierta del mercado de Algeciras (11), con el arquitecto Manuel Sánchez Arcas o el frontón de Recoletos (12), en colaboración con el arquitecto Secundino Zuazo, ambas construidas en 1935, o los graderíos del Hipódromo de la Zarzuela (13), de 1941, junto a los arquitectos Carlos Arniches Moltó y Martín Domínguez, también en Madrid.

Los bombardeos durante la Guerra Civil dejaron irreparablemente dañada la antigua iglesia de San Nicolás del Grau de Gandía debiendo ser derribada en 1953 (14). Dos años después, el nuevo párroco, Juan Miñana, encabezó un movimiento popular destinado a dotar a la población de un espacio religioso digno. Conocidas las excelentes referencias de la iglesia de Pont de Suert, en Lérida, otra de las interesantes obras de Torroja, el padre Miñana, decide proponerle la construcción del nuevo templo. Éste, tras una visita al emplazamiento junto a su hijo José Antonio, asumió el encargo.

El proyecto de la iglesia de San Nicolás lo realizó en colaboración con el arquitecto Gonzalo Echegaray Comba (15) y el ingeniero Jaime Nadal Aixalá (16). El fallecimiento de Torroja en 1961, un año antes de la culminación de las obras, la convierte en su última obra.

La iglesia se encuentra en un extremo del puerto del Grau de Gandía. Desde el exterior el edificio se percibe como un único volumen paralelepipédico, con la cabecera orientada al Este, tal y como establecen los cánones clásicos (Figura 1).

El complejo lo forman la iglesia, la Casa Abadía y el claustro ajardinado con el campanario que une a ambas. La nave principal de la iglesia tiene forma trapezoidal y de $35 \times 10-12 \mathrm{~m}$ en planta con una altura de $13,5 \mathrm{~m}$. confiriéndole al volumen rotundidad y visibilidad.

Adosados a la nave principal se encuentran al Este el Baptisterio, al Sur las capillas laterales, de las que sobresale la capilla del Paso y al Norte el primer ala del claustro (Figura 2). Las fachadas están revestidas con ladrillo caravista con un aparejo con resaltos que junto a las gárgolas de hormigón prefabricado les confiere una textura rítmica (17).

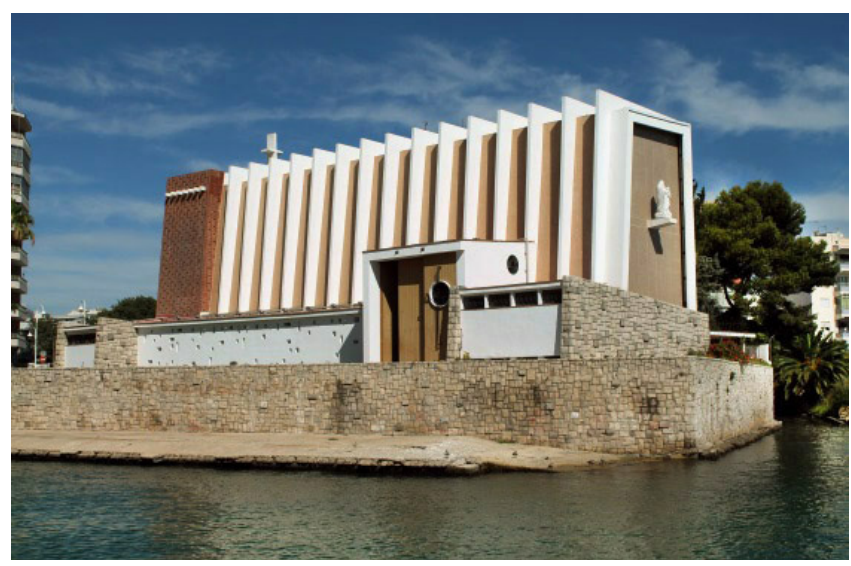

Figura 1. Vista exterior de la iglesia. 


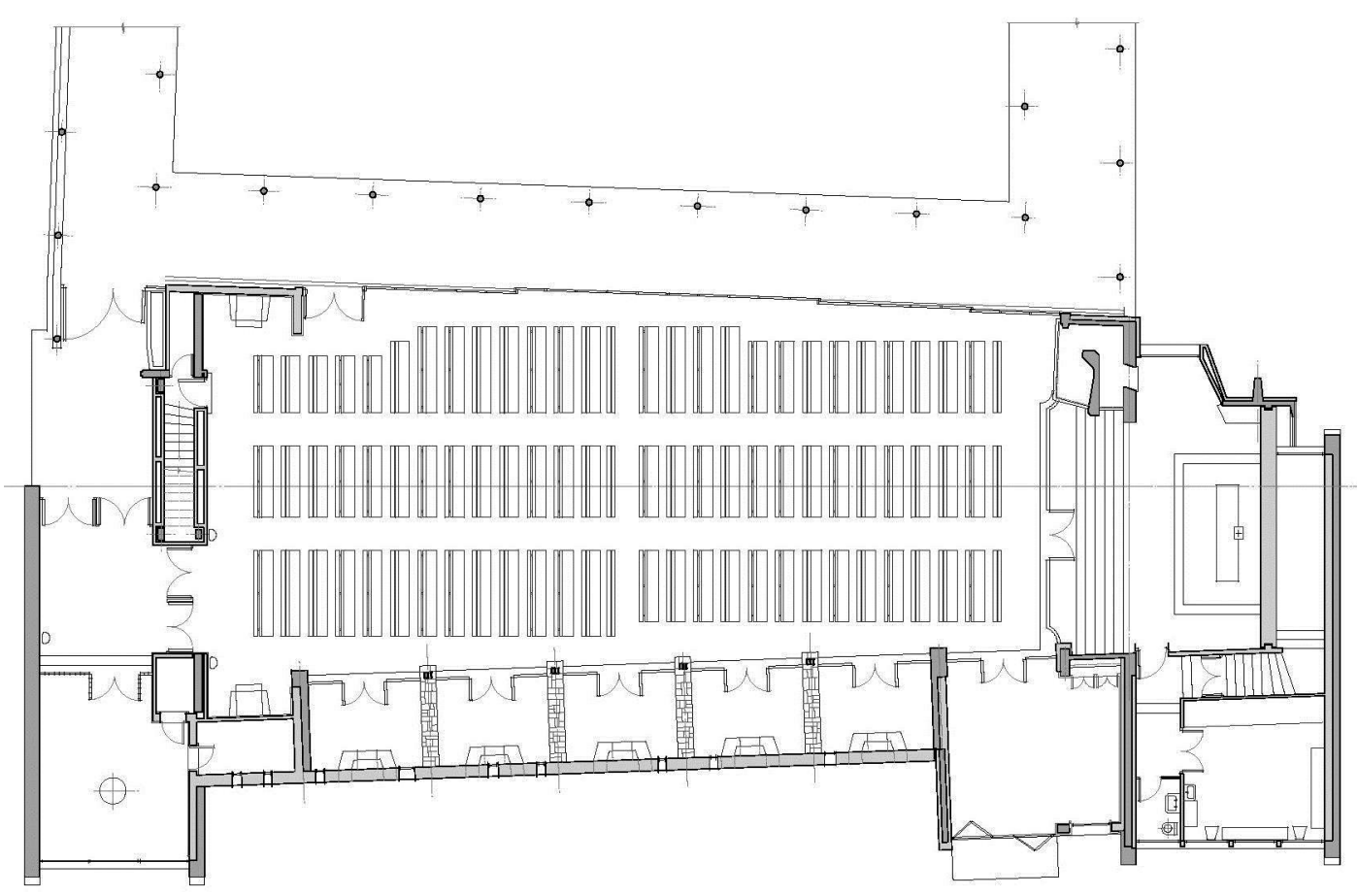

Figura 2. Planta de la iglesia (Echegaray, G. 1959).

Se construye en una época en el que el hormigón armado se ha consolidado en España como principal material para la ejecución de estructuras y se experimenta con nuevos sistemas, que superan el diseño básico configurado mediante pórticos de pilares y vigas. En concreto, se recurre a la utilización de una innovadora solución, mediante la disposición de dos láminas plegadas.

El profesor Cassinello definió de una forma clara este tipo estructural: «las láminas plegadas, definidas por planos que se cortan, ofrecen indudables posibilidades de aplicación al ser capaces de dar un gran canto, pese a su poco espesor, y una gran rigidez transversal, gracias a su forma» (18), (19). Félix Candela las clasificó como el grupo de estructuras laminares que están «sometidas a un régimen mixto de esfuerzos de membrana y de flexión» (20).

Esta geometría de plegaduras tuvo su máximo desarrollo en el siglo xx, concretamente en los años comprendidos entre principios de los 50 y finales de los 60 (21). Según Azpiazu, su decadencia a principios de los años 70, se debe al encarecimiento y complejidad de los encofrados artesanales y por no haber desarrollado un sistema de prefabricación adecuado para su ejecución que permitiera que estas láminas mantuvieran su monolitismo para no perder su condición de cáscara (22).

Torroja proyecta dos láminas plegadas de hormigón, independientes entre sí, apoyadas en los testeros de la edificación, a la manera que Torroja ya utilizó en el Frontón de Recoletos (1)-cubriendo una luz de casi $30 \mathrm{~m}$. y dejando totalmente diáfana la nave principal. La autonomía de las dos láminas plegadas permite generar tres rasgaduras longitudinales: el lucernario de cubierta, el tragaluz sobre las capillas y el acristalamiento al claustro. Cabe destacar la increíble sensación de ingravidez que esta solución confiere a la cubierta de la iglesia (Figura 3).

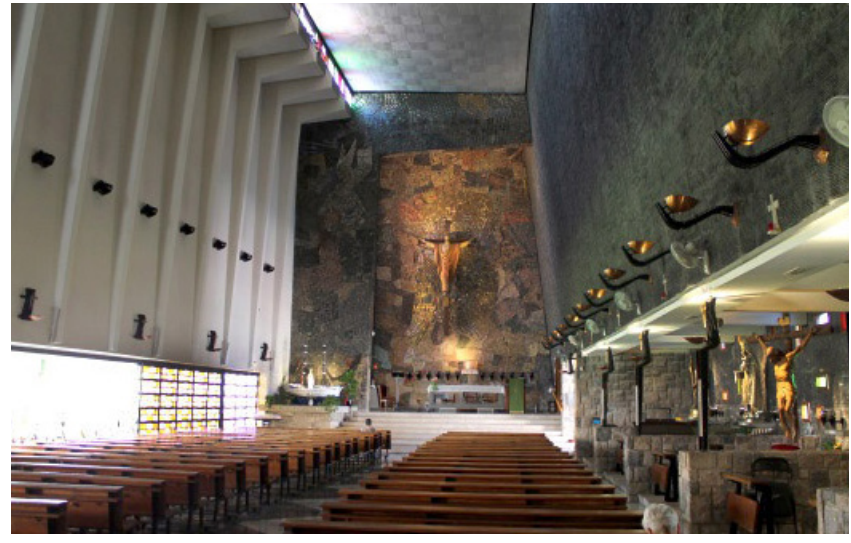

Figura 3. Interior de la iglesia donde se aprecian las tres rasgaduras longitudinales originadas por las dos láminas plegadas.

El proyecto parece estar inspirado en una idea previa de Torroja, plasmada en una serie de dibujos para una modesta iglesia en el campo. En este caso, la cubierta estaba formada por dos láminas de cuarto de cilindro, que recuerda la imagen del Frontón de Recoletos. Al igual que en la iglesia de San Nicolás, las dos cubiertas son completamente independientes entre sí, separadas por un lucernario, en el sentido longitudinal de la nave (Figura 4) (11).

Las láminas de hormigón (Figura 5) adquieren inercia gracias a la altura de los muros (9,5 m el muro Norte y 10,5 m el Sur) y al plegado horizontal en sus extremos. Tienen forma de $\mathrm{Z}$, con el alma formada por las fachadas y el ala superior por la cubierta de la nave. El ala inferior está constituida por la cubierta de las capillas laterales de la lámina Sur y por la cubierta del claustro en la Norte. Sin embargo, la losa inferior de la lámina Sur no se encuentra directamente conectada con el muro, sino que se une únicamente a través de las costillas. Por ello, es frecuente referirse a la forma de esta lámina como una $L$ invertida. 


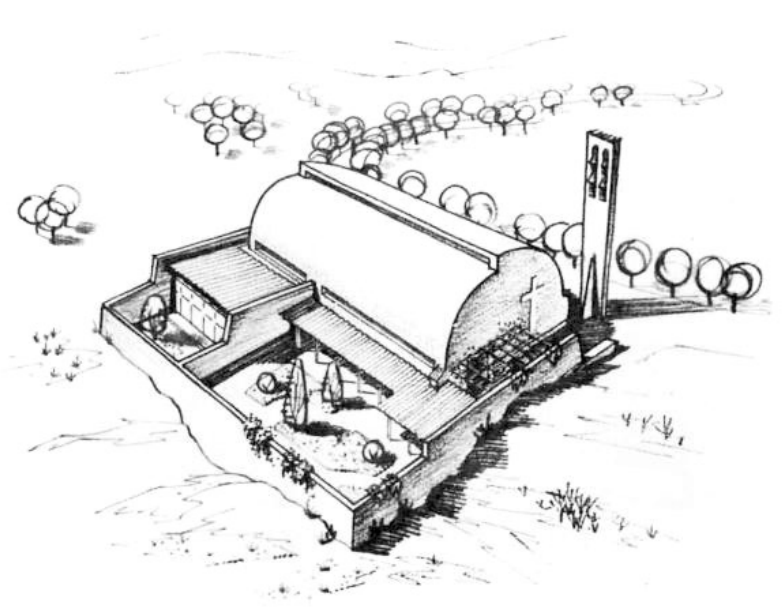

Figura 4. Boceto para una iglesia en el campo (Torroja, E.1958).
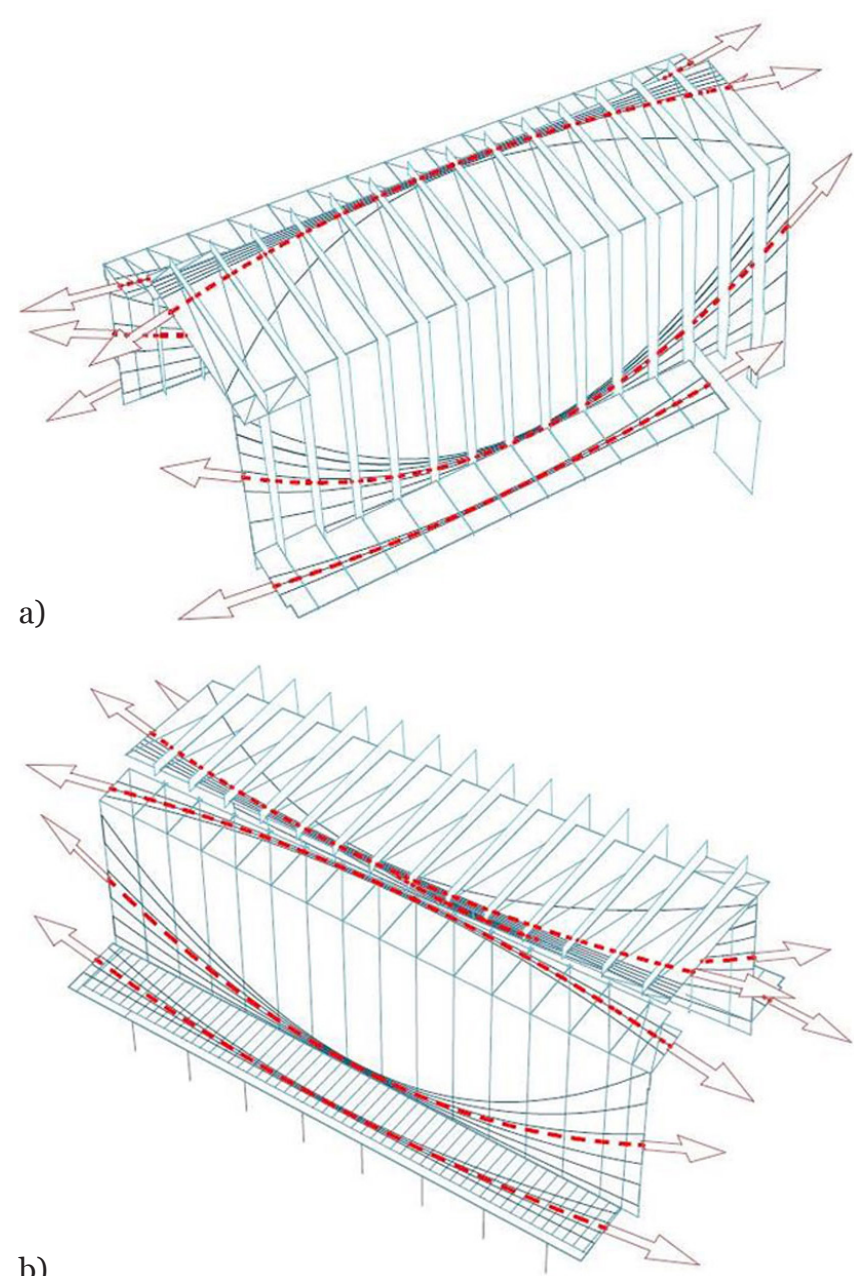

Figura 5. Esquema estructural de las láminas plegadas con la identificación de los pórticos (costillas) y las láminas en Z (losas): a) Lámina Sur, b) Lámina Norte.

El comportamiento estructural de las delgadas láminas es mejorado por Torroja mediante dos soluciones constructivas. Por un lado, dispone una serie de costillas, como elemento de rigidización frente a los esfuerzos de flexión esviada y torsión, causados por la geometría asimétrica y quebrada de las láminas y para evitar los fenómenos de inestabilidad elástica.
Por otro lado, introdujo esfuerzos activos en las losas mediante el postesado de tendones de acero (23). Los situados en la superficie vertical contrarrestan la mayor parte del peso propio, reduciendo los esfuerzos de flexión. Los tendones situados en la cubierta de la nave y de las capillas producen dos fuerzas horizontales de sentidos contrarios que reducen los esfuerzos de torsión (24).

\section{CARACTERIZACIÓN DE LAS LESIONES}

Fernández Cánovas fue supervisor de la primera intervención que se realizó sobre la estructura de la iglesia y a continuación se transcribe la información que facilitó directamente a los autores de este artículo:

«... la intervención estuvo motivada por la fisuración generalizada que presentaban las costillas de la estructura exterior a los 15 años de haberse terminado la obra. Yo era colaborador en el Instituto Eduardo Torroja de Ciencias de la Construcción, trabajando en el Dpto. de Materiales en problemas de patología e investigando en resinas epoxi aplicadas a la construcción. D. Gonzalo Echegaray, me pidió que me ocupara de supervisar la reparación que iba a realizar ACRE (Aplicaciones a la Construcción de Resinas Especiales) en la iglesia de San Nicolás. [...] Las fisuras que aparecían, en vertical, en todas las costillas coincidían con la posición de las barras de esquina y estaban motivadas por la expansión provocada por la corrosión de las mismas. Se hizo un análisis con fenolftaleína y se observó una profundidad de carbonatación que superaba el espesor del recubrimiento. Se realizó un análisis de cloruros, tomando unas muestras, personalmente, y acompañado por mí, D. Pablo García de Paredes [...], y los resultados nos indicaron un valor de cloruros tres veces superior a los límites permitidos. Hablando con personal de Gandía que había trabajado en la construcción de la iglesia nos confirmaron que la arena que se había empleado en la construcción era de playa y que no se había lavado con agua dulce. Esto confirmaba lo que ya habíamos deducido al observar trozos de cascaras de moluscos, especialmente almejas, en el hormigón de los recubrimientos que habíamos eliminado.

La intervención consistió en eliminar los recubrimientos, que eran escasos, limpiar las barras mecánicamente con cepillo de alambre, darles una pintura protectora de epoxi-zinc y posteriormente rehacer el recubrimiento con un hormigón adecuado de cemento portland.

Recuerdo perfectamente que, al finalizar la obra, informe en una reunión que tuvimos, a Gonzalo Echegaray y a mi jefe, Francisco Arredondo, que ésta no era una solución definitiva y que en un futuro, más o menos próximo, volverían a aparecer problemas, pues no sabíamos la situación de los cables de postensado, ni de otros elementos interiores sobre los que no habíamos intervenido».

En 1996, transcurridos 35 años de su construcción, la iglesia de San Nicolás volvió a presentar una serie de daños. El arquitecto Agustín Gabriel, en calidad de asesor arquitectónico de la parroquia, encargó el análisis y la evaluación de las lesiones a los laboratorios Intemac.

Fruto de este encargo se produjo la primera visita al templo del ingeniero de caminos Enrique González Valle, director de Intemac, el 31 de mayo de 1996. El resultado de esta primera 
inspección fue la redacción del informe preliminar de fecha 25 de julio de 1996, en el que se documentaron las lesiones, se apuntaron las posibles causas que las originaban, se realizó una evaluación de su trascendencia y se establecieron las recomendaciones oportunas.

Dichas recomendaciones derivaron en una serie de campañas de medidas y de ensayos, sobre unas probetas testigo extraídas previamente. Los resultados se recogieron en un segundo informe de los laboratorios Intemac, fechado el 7 de agosto de 1998.

El informe final incluye los mencionados informes, a modo de anejos, juntamente con los croquis de los daños, los partes de extracción y ensayo a compresión de las probetas testigo de hormigón, los resultados de las campañas de medidas y un anejo fotográfico. De dicho informe, fechado el 9 de febrero de 1999, se desprende la siguiente información:

\subsection{Relación daños estructurales}

\subsubsection{Pórticos-costillas}

En la fachada Sur (Figura 6a), se observaron fisuras en la cara superior de las vigas horizontales de la cubierta, siguiendo el trazado de las armaduras superiores que presentaban corrosión leve o casi inexistente, con un espesor de recubrimiento de hormigón, entre $10 \mathrm{~mm}$ y $20 \mathrm{~mm}$. Los tramos verticales del pórtico tenían una fisuración importante, con anchos entre 0,5 y $5 \mathrm{~mm}$, marcando la posición de las armaduras principales.

En la fachada Norte, los pórticos son interiores, no expuestos directamente al ambiente exterior, y no presentaban fisuración visible, salvo en el tramo de cubierta de los pórticos de la esquina Noreste del edificio, en la que se observaron unos daños leves.

\subsubsection{Losas de las láminas plegadas}

La losa plegada ubicada en la zona Sur presentaba fisuración importante en el frente de cubierta, a lo largo del lucernario superior. En algunas zonas se observó el desprendimiento total del recubrimiento (Figura 6b).

No obstante, se observó que en las zonas comprimidas por la acción del postesado, la fisuración de las láminas era menor, reduciendo, en consecuencia, la exposición de las armaduras al ambiente exterior.

\subsubsection{Pilares y cubierta del claustro}

Prácticamente todos los elementos presentaban daños por corrosión de las armaduras, desde la base hasta una altura que varía de $0,13 \mathrm{~m}$ a 1,22 $\mathrm{m}$. Además dos pilares en la esquina Suroeste del claustro mostraban fisuras horizontales en cabeza, otro en la misma zona presentaba una fisura sinusoidal notable, que seccionaba completamente el pilar y finalmente un pilar situado en la cara Este del claustro presentaba una rotura horizontal importante en la unión con la losa superior. Además, la losa superior presentaba fisuras con ancho variable entre 0,5 y $2 \mathrm{~mm}$.

\subsubsection{Campanario}

Debido a la corrosión de las armaduras presentaba en su base fisuras verticales con anchura máxima de $5 \mathrm{~mm}$. La losa superior, situada en los dos huecos rectangulares de paso presentaba también daños importantes por corrosión, llegando a la pérdida total del recubrimiento de las armaduras.

\subsubsection{Otras localizaciones}

La mayoría de elementos ornamentales de hormigón armado (marco de la fachada Este, ménsulas y la cruz) presentaban daños importantes causados por la corrosión de las armaduras, llegando a pérdidas totales del recubrimiento en determinados puntos.

Algunos cerramientos y soleras, especialmente del muro Sur de las capillas laterales, presentaban daños importantes producidos por asientos diferenciales. Cabe mencionar que su cimentación es de tipo superficial, sobre rellenos, y la del resto de la estructura por pilotaje a mayor profundidad.
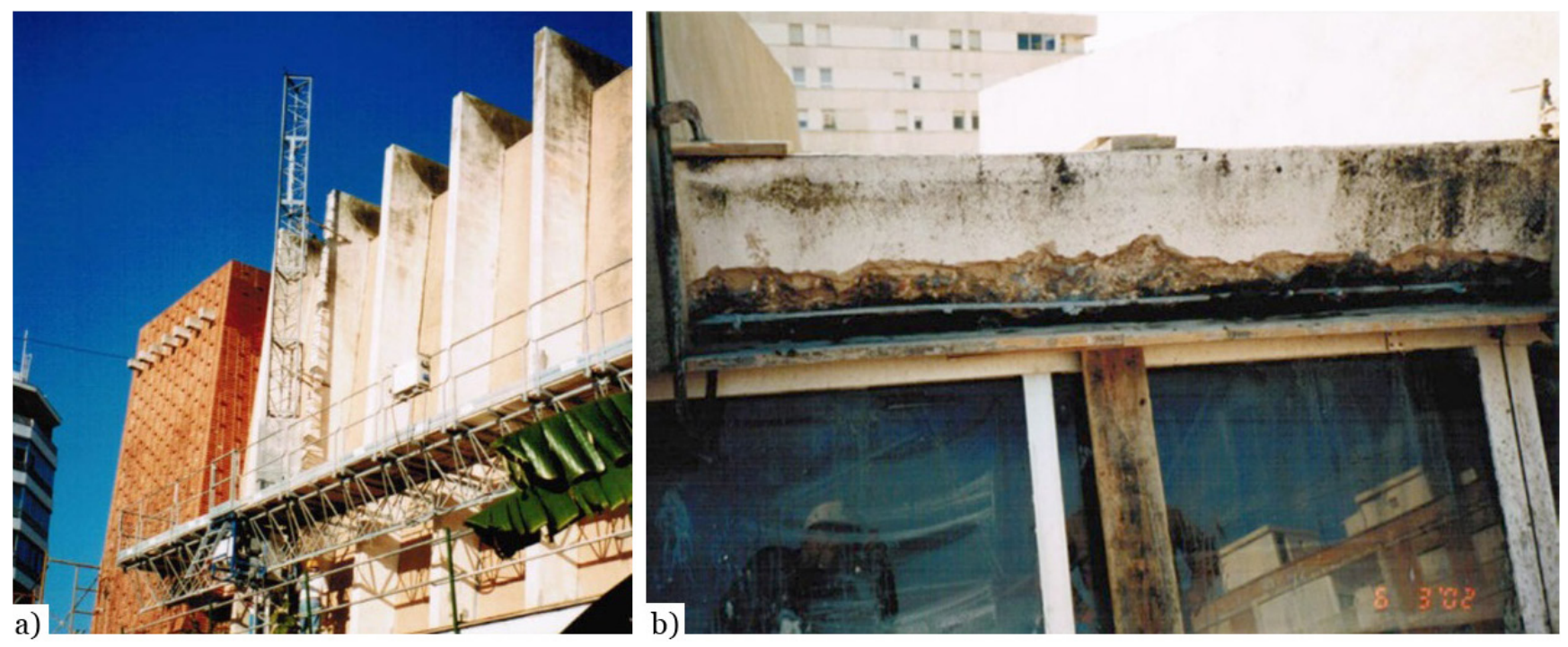

Figura 6. Estado que presentaba los elementos estructurales de la fachada Sur:

a) Pórticos-costillas, b) Detalles de la pérdida de recubrimiento de la losa. (Lafuente, I. 2002). 


\subsection{Relación de daños en elementos constructivos}

Se observaron filtraciones de agua de lluvia, manchas de humedad y desconchones de la pintura en las cubiertas del claustro y del acceso. Eran debidos al deterioro de la impermeabilización de cubiertas planas sobre las losas de hormigón y a las filtraciones del sistema de evacuación de pluviales afectadas por movimientos diferenciales en la estructura.

En todas las fachadas se habían producido desprendimientos parciales de los acabados exteriores y de los revestimientos vitraicos, por fallos en la adherencia con el soporte. También se observaban filtraciones de agua a través de los muros de ladrillo caravista.

El paramento interior del muro Sur, de revoco raspado, se encontraba afectado por las filtraciones y la polución, humo de velas, etc. El del muro Norte, de mortero poroso, estaba afectado por filtraciones, desprendimientos y abolladuras.

Se constató la existencia de pavimentos cerámicos degradados en el acceso y claustro, debido a la humedad procedente de los jardines. También se habían producido filtraciones de agua en el semisótano y humedades por capilaridad en el cerramiento de las capillas. El pavimento interior de terrazo bicolor presentaba lesiones leves y generalizadas de decoloración y porosidad debido a humedades por capilaridad.

Las carpinterías de acero laminado presentaban un avanzado deterioro por corrosión debido a la pérdida de protección por falta de mantenimiento.

Los falsos techos aparecían abombados y mostraban daños por filtraciones de agua.

\subsection{Relación de daños en otros elementos}

Se observaron barreras arquitectónicas que reducían la accesibilidad al edificio y otras que producían incomodidades en la circulación del paso en la fachada Sur, durante las celebraciones religiosas.

Se detectó la presencia de cerramientos impropios en el claustro.

La instalación eléctrica se encontraba obsoleta, así como los sistemas de iluminación. Las luminarias primitivas mostraban mal estado de conservación.

\section{ENSAYOS Y PRUEBAS REALIZADOS}

A continuación se presentan los resultados obtenidos de los ensayos realizados, que también quedan reflejados de forma resumida en la Tabla 1.

Campaña de medidas de potencial de corrosión, mediante electrodo de cobre-sulfato de cobre y voltímetro de $1 \mathrm{mV}$ de apreciación, según ASTM C-876 (25). Se realizó en 16 zonas de pórticos y pilares del claustro, efectuando mediciones del potencial eléctrico sobre el hormigón en diez puntos de la barra seleccionada, separados aproximadamente $150 \mathrm{~mm}$ entre sí.

Campaña de medidas de resistividad del hormigón, mediante electrodo a cuatro hilos. Se analizaron 16 zonas de pórticos y pilares del claustro, midiendo la resistividad en cinco puntos de cada zona.

Ensayo a compresión y determinación de la densidad y porosidad del hormigón. Se extrajeron 6 probetas testigo de hormigón endurecido destinadas a un ensayo de compresión y otras 3 probetas destinadas a ensayos químicos según normas UNE 83302 (26), UNE 83303 (27), UNE 83304 (28). Consultando la instrucción HA-61 (29), coordinada por Torroja y de carácter voluntario, la resistencia característica mínima debía ser mayor o igual a $130 \mathrm{~kg} / \mathrm{cm}^{2}$, con aceros lisos, y 170 con los de alta adherencia, valores inferiores a los obtenidos en las probetas ensayadas, con un valor mínimo de $235 \mathrm{~kg} / \mathrm{cm}^{2}$.

La porosidad del hormigón se determinó sobre las 6 probetas testigo según la ASTM C-642 (30).

Determinación de la profundidad de carbonatación en las 6 probetas testigo extraídas, mediante impregnación alcohólica de fenolftaleína. La prueba se realizó en todos los puntos de extracción de las probetas testigo y en 10 puntos adicionales, siguiendo los criterios de la norma UNE 112011 (31) y con una apreciación de $1 \mathrm{~mm}$. Consultando el informe de evaluación, se desprende que las profundidades de carbonatación, en general, no excesivas para la edad de la estructura, con valores que oscilan varían entre 2 y $7 \mathrm{~mm}$.

Determinación del perfil de cloruros en 3 probetas testigo obtenidas de fragmentos del recubrimiento desprendido y en otro obtenido por golpeo en uno de los pilares del claustro. Siguiendo la norma UNE 112010 (32) se determinó el residuo en ácido salicílico en muestras análogas para expresar los contenidos de cloruros respecto a la dosificación del cemento. Del informe de evaluación se concluye que los contenidos de ión $\mathrm{Cl}^{-}$, son en algún caso superior al valor límite umbral de riesgo de corrosión (o,4 \% en peso de cemento).

Determinación de la permeabilidad, sobre 3 probetas testigo, según UNE 83309 (33).

Tras la eliminación del exceso de agua de la base se realizó el ensayo a tracción indirecta según UNE 83306 (34), perpendicularmente a la cara sometida a presión de agua y se determinaron las profundidades de penetración media y máxima. Del análisis de resultados se deduce que los valores de penetración de agua bajo presión son muy superiores a los límites admitidos para considerar al hormigón suficientemente impermeable (profundidad máxima $\leq 50 \mathrm{~mm}$; profundidad media $\leq 30 \mathrm{~mm}$ ).

A continuación, la Tabla 1 recoge un resumen de todas las pruebas y ensayos.

\section{CAUSAS POSIBLES Y ACTUACIONES PROPUESTAS}

La causa de los daños estructurales era fundamentalmente la corrosión de las armaduras próximas a los paramentos expuestos a la agresión ambiental. Dicha corrosión tiene tres componentes básicos:

Escasa o nula protección del mortero por su contenido en cemento y porosidad. 
Tabla 1. Resumen de las pruebas y ensayos efectuadas. (Elaboración propia a con datos de los informes de Intemac).

\begin{tabular}{|c|c|c|c|c|c|c|c|c|c|c|c|c|c|c|}
\hline \multirow{2}{*}{ Ubicación } & \multirow{2}{*}{$\begin{array}{c}\text { Elemento } \\
\text { estructural }\end{array}$} & \multirow{2}{*}{$\begin{array}{l}\text { Valor } \\
\text { máximo } \\
\text { potencial } \\
\text { corrosión } \\
\text { (mv) }\end{array}$} & \multirow{2}{*}{$\begin{array}{c}\text { Valor } \\
\text { máximo } \\
\text { resistividad } \\
(\mathrm{k} \Omega \times \mathrm{cm})\end{array}$} & \multirow{2}{*}{$\begin{array}{l}\text { Profundidad } \\
\text { de carbona- } \\
\text { tación } \\
(\mathrm{mm})\end{array}$} & \multirow{2}{*}{$\begin{array}{l}\text { Recubri- } \\
\text { miento } \\
\text { armaduras } \\
\quad(\mathrm{mm})\end{array}$} & \multicolumn{2}{|c|}{$\begin{array}{c}\text { Diámetro } \\
\text { armaduras } \\
(\mathrm{mm})\end{array}$} & \multirow{2}{*}{$\begin{array}{l}\text { Resisten- } \\
\text { cia a } \\
\text { compresión } \\
\left(\mathrm{Kp} / \mathrm{cm}^{2}\right)\end{array}$} & \multicolumn{2}{|c|}{$\begin{array}{c}\text { Contenido } \\
\text { en } \\
\text { cloruros } \\
(\%)\end{array}$} & \multirow{2}{*}{$\begin{array}{c}\text { Porosidad } \\
\text { (\%) }\end{array}$} & \multirow{2}{*}{$\begin{array}{l}\text { Densidad } \\
\text { aparente } \\
\left(\mathrm{Kg} / \mathrm{dm}^{3}\right)\end{array}$} & \multicolumn{2}{|c|}{$\begin{array}{l}\text { Profundidad } \\
\text { permeabi- } \\
\text { lidad } \\
\text { al agua } \\
\text { (mm) }\end{array}$} \\
\hline & & & & & & 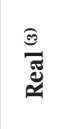 & 즐 & & 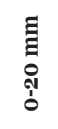 & 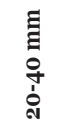 & & & 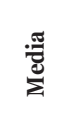 & 范 \\
\hline \multirow{7}{*}{$\begin{array}{l}\text { Fachada } \\
\text { Sur vertical }\end{array}$} & Pórtico 1 & -180 & 89,5 & $49^{(1)}$ & $44^{(1)}$ & 25,0 & 25,0 & 382 & 0,39 & 0,87 & 13,1 & 2,30 & 84 & 90 \\
\hline & Pórtico 2 & -175 & 78,6 & $54^{(1)}$ & $52^{(1)}$ & 25,0 & 25,0 & & & & & & & \\
\hline & Pórtico 4 & -100 & 82,4 & $47^{(1)}$ & $45^{(1)}$ & 25,0 & 25,0 & 239 & 0,17 & 0,26 & 17,8 & 2,15 & & \\
\hline & Pórtico 5 & -478 & 98,0 & $55^{(1)}$ & $50^{(1)}$ & 23,1 & 25,0 & & & & & & & \\
\hline & Pórtico 6 & -72 & 86,5 & $20^{(1)}$ & $35^{(1)}$ & 25,0 & 25,0 & & & & & & & \\
\hline & Pórtico 8 & -396 & 80,4 & $45^{(1)}$ & $47^{(1)}$ & 25,0 & 25,0 & 264 & & & 15,1 & 2,23 & 106 & 118 \\
\hline & Pórtico 9 & -562 & 76,5 & $>55^{(1)}$ & $55^{(1)}$ & 25,0 & 25,0 & & & & & & & \\
\hline $\begin{array}{l}\text { Fachada } \\
\text { Norte vertical } \\
\text { interior }\end{array}$ & Losa Z & - & 137,0 & $45^{(2)}$ & $-{ }^{(1)}$ & - & - & 235 & & & 11,1 & 2,36 & & \\
\hline $\begin{array}{l}\text { Fachada } \\
\text { Sur cubierta }\end{array}$ & Pórtico 0 & -180 & 102,0 & $28^{(1)}$ & $22^{(1)}$ & 26,0 & 25,0 & & & & & & & \\
\hline \multirow{4}{*}{$\begin{array}{l}\text { Fachada Sur } \\
\text { cubierta }\end{array}$} & Pórtico 3 & -230 & 109,0 & $27^{(1)}$ & $40^{(1)}$ & 25,5 & 25,0 & 268 & & & 12,2 & 2,32 & & \\
\hline & Pórtico 6 & -94 & 90,4 & $35^{(1)}$ & $52^{(1)}$ & 25,0 & 25,0 & 239 & & & & & & \\
\hline & Pórtico 8 & & & & & & & & & & 14,9 & 2,25 & & \\
\hline & Pórtico 11 & -76 & 95,6 & $33^{(1)}$ & $70^{(1)}$ & 25,0 & 25,0 & & 0,29 & 0,36 & & & 113 & 118 \\
\hline \multirow{4}{*}{ Claustro } & Pilar A & -344 & 24,5 & $>20$ & 16 & 8,1 & 8,0 & & & & & & & \\
\hline & Pilar D & -456 & 26,7 & $>40$ & 20 & 8,3 & 8,0 & & 0,39 & 0,39 & & & & \\
\hline & Pilar F & -484 & 34,2 & $>20$ & 15 & 8,4 & 8,0 & & & & & & & \\
\hline & Pilar G & -382 & 42,6 & $>20$ & 13 & 8,4 & 8,0 & & & & & & & \\
\hline
\end{tabular}

Notas:

(1) Incluye espesor de la capa superficial de mortero (entre 15-25 mm).

(2) El paramento de hormigón estaba revestido por una capa de material aislante de $15 \mathrm{~mm}$ de espesor.

(3) Medido con pie de rey tras practicar una cala en el elemento estructural.

Escasos espesores de recubrimiento y elevada densidad de armado que dificulta el hormigonado homogéneo.

Ambiente de alta agresividad, junto al mar, con elevada salinidad y presencia de cloruros que, como se ha comentado en su ensayo de determinación, no sólo procedía de la aportación del ambiente exterior, sino que se encontraba presente en la propia masa del hormigón, debido a la utilización de arena no lavada.

La presencia de cloruros y carbonatación de la protección de las armaduras, condujo a su corrosión y a la fisuración de los recubrimientos, acelerando el proceso de corrosión por aumentar la exposición. En el caso de los pilares del claustro este proceso de corrosión se vio también acelerado por el ascenso de humedades por capilaridad al elevar la concentración de cloruros con el aporte de humedad y desecación superficial.

Los deterioros observados en los cerramientos y soleras de las capillas laterales se deben a los asientos diferenciales entre la cimentación de tipo superficial sobre rellenos y la del resto de la estructura realizada por pilotaje a mayor profundidad.

En los elementos no estructurales, como pavimentos y cerrajería, las causas de los daños se asocian a la falta de mantenimiento en el edificio.
La seguridad estaba condicionada por dos factores básicos: la pérdida de sección de las armaduras por corrosión y la pérdida de adherencia de tales armaduras con el hormigón.

Los daños observados y los datos de los análisis posteriores evidencian que la seguridad no se encontraba comprometida a corto plazo. No obstante, los problemas graves de durabilidad hacían necesaria una intervención inmediata para impedir un progreso acelerado de los daños.

Estas circunstancias eran distintas en los pilares de claustro, con las bases muy deterioradas. A pesar de que nominalmente las solicitaciones sobre los pilares eran muy reducidas, el mal estado de armaduras y hormigón hacían urgente la intervención.

\section{EL PROYECTO DE INTERVENCIÓN}

Siguiendo sus recomendaciones del informe final de Intemac, la parroquia promueve las obras de rehabilitación integral, coincidiendo con la celebración del 50 aniversario en 2012.

El objetivo del proyecto de intervención es la reparación o sustitución de los elementos dañados y la renovación de las instalaciones en estado de obsolescencia. Así mismo se pretendían eliminar elementos impropios y renovar acabados. 
El proyecto y dirección de obra se encargó al arquitecto Ignacio Lafuente. La dirección de la ejecución material de las obras se encomendó al arquitecto técnico José Bernardo Rodríguez Ripoll.

\subsection{Fases del proceso de rehabilitación}

La rehabilitación se acometió en tres fases, tanto en proyecto como en ejecución.

Fase 1 (2002): Proyecto de Ejecución visado en el Colegio Territorial de Arquitectos de Valencia, el 16 de noviembre de 2001. Las obras se adjudicaron a PINTURAS TEP S.L., especializado en reparaciones de estructura de hormigón. Durante esta fase se acometieron las siguientes labores:

- Intervenciones estructurales en cubierta de la nave y fachadas Este y Sur.

- Renovación del sistema de evacuación de pluviales de fachada Sur.

- Reposición de revestimientos vitraicos de las fachadas Sur y Este.

- Eliminación de barreras arquitectónicas con la construcción de una rampa de acceso a la iglesia.

Fase 2 (2005): Proyecto de Ejecución visado en el Colegio Territorial de Arquitectos de Valencia, el 23 de Junio de 2003. De nuevo adjudicado a PINTURAS TEP S.L. Se realizaron los siguientes trabajos:

- Intervención sobre elementos estructurales: estructura del claustro y del campanario.

- Impermeabilización de losas del claustro y acceso.

- Reposición de revestimientos vitraicos sobre el paramento de ladrillo de la Fachada Norte y de fachadas al claustro.

- Sustitución de pavimentos cerámicos de acceso y claustro.

- Eliminación elementos procedentes de intervenciones impropias en el claustro.
- Tratamiento hidrófugo de paramentos de ladrillo caravista

- Restauración de la vidriera del altar en la fachada Norte.

Fase 3 (2005-2006): Proyecto de Ejecución visado en el Colegio Territorial de Arquitectos de Valencia, el 12 de noviembre de 2004. Se adjudicó al contratista TALENS S.L. Incluyó las siguientes obras:

- Renovación, impermeabilización y colocación de pavimentos exteriores en la fachada Sur.

- Eliminación mediante una acera del ajardinamiento al pie del cerramiento de las capillas en la fachada Sur.

- Sustitución de pavimento interior y reposición de paramentos interiores de la nave.

- Reposiciones de placas de falso techo y renovación de la escayola continua.

- Renovación funcional de la instalación eléctrica.

- Restauración de luminarias primitivas del templo y sustitución del resto.

- Instalación de aseo accesible al público.

- Instalación de red de riego por goteo y aspersores y reposición del ajardinamiento.

\subsection{Intervención sobre elementos estructurales}

El proyecto de intervención, sigue las recomendaciones presentes en el Informe de Intemac de 9 de febrero de 1999. Se actuó sobre la cubierta de la nave y las fachadas Este y Sur y en la estructura del claustro y del campanario.

El proceso de reparación y los sistemas utilizados fueron los siguientes:

- Saneado del hormigón dañado, mediante medios mecánicos o chorro de agua de alta presión (Figura 7a). En el caso de los pilares del claustro esta fase, se realizó tras el apeo de la losa de hormigón.
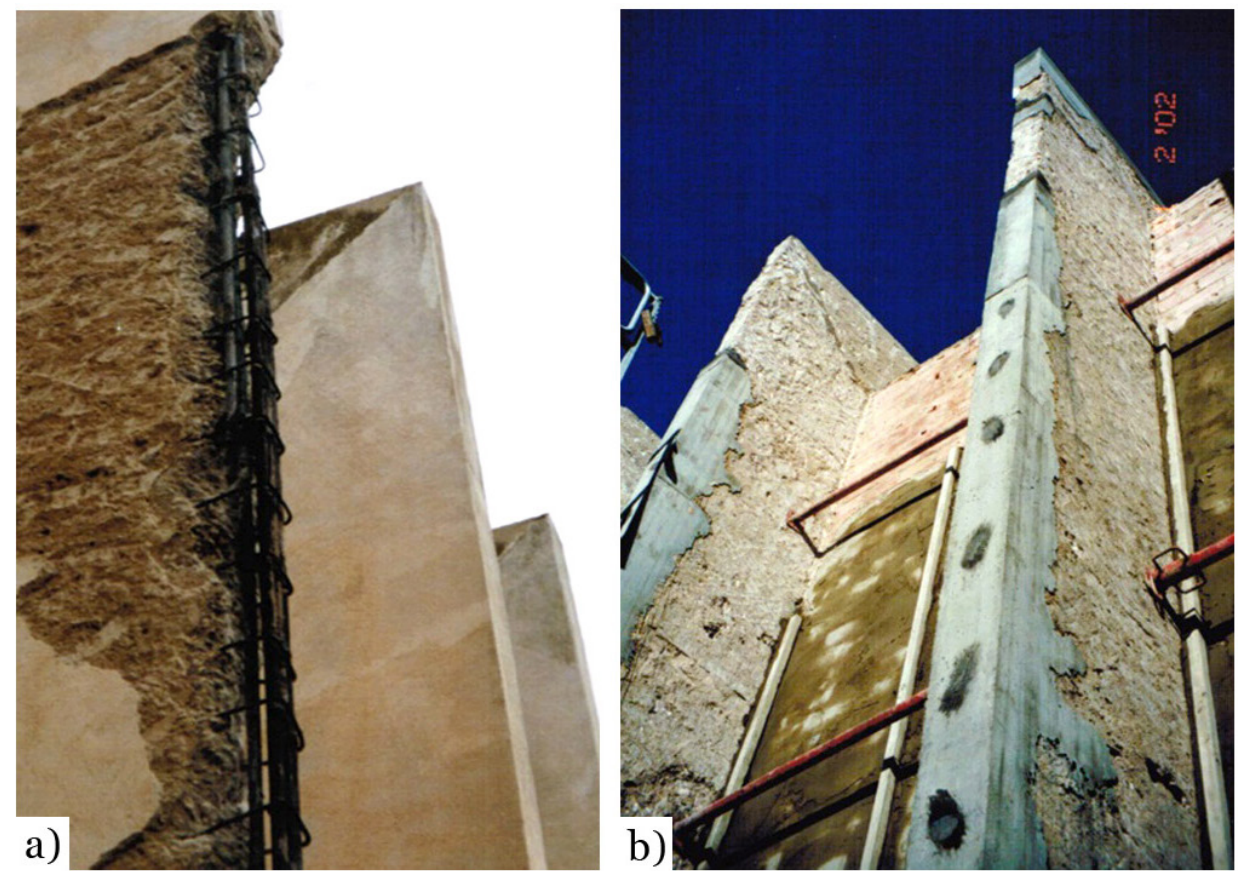

Figura 7. Intervención en los pórticos-costillas de la fachada Sur: a) Saneado, b) Reposición del hormigón. (Lafuente, I. 2002) 
- Limpieza de las armaduras del hormigón: Mediante chorro de arena de sílice para eliminar los óxidos procedentes de la corrosión. Posteriormente, tanto las armaduras como el hormigón sano se limpiaron mediante chorro de agua a media presión.

- Pasivación de las armaduras: Tras su limpieza se aplicaron dos capas de pintura de revestimiento pasivante, en un intervalo inferior a $4 \mathrm{~h}$, enarenando la segunda capa. La pintura utilizada fue LEGARAN de Basf.

- Reposición del hormigón afectado (Figura 7b): Mediante aplicación de mortero de cemento modificado con polímeros, aplicado según el caso a mano, por proyección, o gunitado, en capas sucesivas menores de $20 \mathrm{~mm}$, previo humedecido del soporte. Para las vigas horizontales se usó BETTOGROUT 150 de Basf, que por su elevada fluidez se coloca por vertido en un encofrado, garantizando la adherencia con el hormigón horizontal. El mortero se puede aglomerar con cemento portland, consiguiendo un efecto protector de las armaduras gracias a la basicidad del cemento. Las reposiciones verticales se realizaron con un mortero de cemento y arena con el aditivo PCI-EMULSIÓN de Basf. Dicho aditivo mejora la impermeabilidad, la adherencia con el hormigón base y resistencia mecánica, disminuyendo la fisuración.

\subsection{Intervención sobre elementos constructivos}

- Impermeabilización de las cubiertas planas sobre las losas de hormigón y renovación del sistema de evacuación de pluviales. Se tuvo especial atención al borde perimetral con la renovación del goterón y la renovación de los sumideros.

- Tratamiento hidrófugo de paramentos de ladrillo caravista. Se aplicaron dos capas de MASTERSEAL $325 \mathrm{E}$ de Basf. Este revestimiento es un monocomponente de base acrílica que resulta muy impermeable al agua y anhídrido carbónico y, sin embargo, es permeable al vapor de agua, permitiendo la transpiración de la estructura.

- Intervención sobre los revestimientos cerámicos. Los acabados cerámicos se adhirieron mediante FLEXMORTEL de Basf, un adhesivo se C2TE, según UNE EN 12004 (35), acreditando una adherencia mínima de $1 \mathrm{~N} / \mathrm{mm}^{2}$, seguro y durable. Las juntas se rellenaron con FLEXFUGE de Basf, deformable e impermeable. De este modo garantiza la continuidad en la impermeabilidad de las piezas cerámicas y permite movimientos como los producidos por las variaciones térmicas.

- Renovación e impermeabilización de pavimentos exterio-

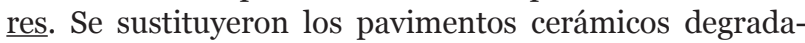
dos de acceso y claustro. Se eliminó el ajardinamiento al pie del muro del cerramiento de las capillas en la fachada Sur, mediante una acera realizada con adoquín y hormigón impreso sobre lámina impermeable. Con esta última intervención se consiguió una cómoda circulación del paso procesional desde la capilla y se evitaron las humedades en semisótano, sacristía y almacén, así como el acceso de la humedad por capilaridad en la base del muro.

- Restauración de vidrieras. Consistió en el desmontaje de vidrios, limpieza de óxidos en la carpintería y aplicación de protección con pintura al zinc-fosfato, reposición de vi-

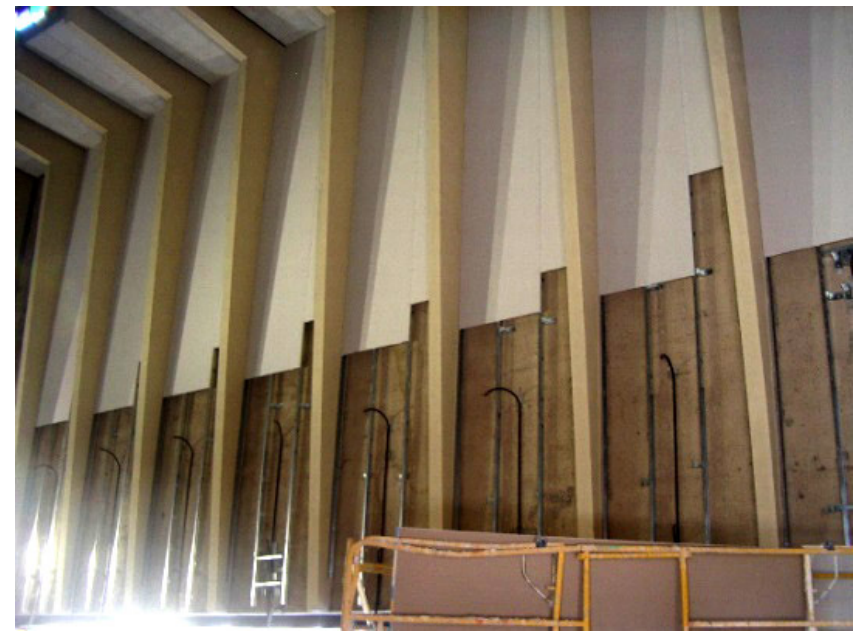

Figura 8. Trasdosado de placas de yeso en el interior de la fachada Norte (Lafuente, I. 2006)

drios y rejuntado con masilla de poliuretano espatulable. La corredera acristalada de acero que separa la nave del claustro fue sustituida por otra de idéntico diseño con entramado galvanizado, tratada con epoxi al zinc fosfato y las vidrieras fueron repuestas.

- Reposición de pavimento interior deteriorado por el uso y por humedades de capilaridad. Se sustituyó por baldosas de granito blanco cristal nacional y gris ibérico portugués con igual dimensión y despiece que los originales, pero con una capa aislante intermedia. El escalón de piedra del borde del altar, dañado tras la eliminación de la cancela cuando se adaptó a la reforma litúrgica del Concilio Vaticano II, fue sustituido por otro de similares características.

- Intervención en los acabados interiores de la nave. Los paramentos de la zona Sur se limpiaron y pintaron. Los paramentos de la zona Norte se doblaron con sistema de entramado y placas de yeso laminado como trasdosado (Figura 8). Se sustituyeron las placas de falso techo abombadas por las filtraciones de agua y se renovaron los falsos techos continuos.

\subsection{Intervención sobre otros elementos}

- Eliminación de barreras arquitectónicas. Se mejoró la accesibilidad de la iglesia con la construcción de la rampa de acceso en la entrada principal.

- Renovación instalación de iluminación. Se restauraron las luminarias primitivas de diseño específico para el templo y sustituyeron el resto, diseñándose una iluminación exterior de las fachadas de carácter monumental.

- Eliminación de cerramientos impropios introducidos en intervenciones anteriores en el claustro

\section{CONCLUSIONES}

El presente trabajo ha mostrado los daños que presentaba la iglesia de San Nicolás, 35 años después de su construcción y el proyecto y obras realizadas para devolver al edificio su carácter original. 
La inspección y evaluación de los daños realizada por los laboratorios Intemac muestran que los daños más importantes se han producido en la estructura de hormigón armado.

El paso del tiempo y, especialmente, la situación del edificio en un ambiente agresivo provocaron la corrosión de las armaduras y la fisuración del hormigón. La corrosión se vio acelerada por la ausencia de mantenimiento, la presencia de cloruros en la propia masa del hormigón y los escasos recubrimientos de las armaduras.

Los elementos estructurales más dañados son exteriores, como las costillas o los soportes del claustro. En las láminas sólo se detectaron daños puntuales lo que revela que los tendones postesados, al mantener el hormigón comprimido $\mathrm{y}$, por tanto con poca o nula fisuración, han preservado sus armaduras de la corrosión, a pesar de los escasos recubrimientos. Así pues, se pone de manifiesto que el postesado del hormigón es un buen recurso para incrementar la durabilidad de la estructura, especialmente en ambientes agresivos.
Se ha expuesto el proyecto original y posteriores obras de rehabilitación. Además de la reparación de los elementos estructurales y constructivos dañados y la sustitución de diversos revestimientos, se aprovecharon las obras de rehabilitación para renovación de las instalaciones que habían quedado obsoletas, eliminar elementos impropios y mejorar la accesibilidad.

Finalmente cabe indicar que el templo, un reconocido ejemplo de la arquitectura contemporánea española, ha podido recuperar su carácter original justo para la celebración del 50 aniversario de su construcción.

\section{AGRADECIMIENTOS}

Archivo del Instituto de Ciencias de la Construcción Eduardo Torroja.

Archivo Torroja de CEHOPU de CEDEX.

Arxiu Històric de Gandia.

Al padre D. Juan Miñana Pavía, fallecido el 13/12/2013, y a los demás sacerdotes y personal de la iglesia.

A D. Ignacio Lafuente Niño, arquitecto autor del proyecto y dirección de obra de la rehabilitación.

\section{REFERENCIAS}

(1) Fernández, J.A., Navarro J.R. (1999). Eduardo Torroja. Ingeniero, Engineer. Madrid: Pronaos.

(2) Jordá Such, C. (2010). Introducción: Los Equipamientos en España. En Equipamientos I: Lugares públicos y nuevos programas 1925-1965, (pp. 11-30). Barcelona: Fundación Caja de Arquitectos. Registro DOCOMOMO Ibérico.

(3) Jordá Such, C. (2010). Capítulo: Comunidad Valenciana. En Equipamientos I: Lugares públicos y nuevos programas 1925-1965, (pp. 249-281). Barcelona: Fundación Caja de Arquitectos. Registro DOCOMOMO Ibérico.

(4) Jordá Such, C. (Ed). (2002). Eduardo Torroja. La vigencia de un legado. Valencia: Universidad Politécnica de Valencia.

(5) Fernández, J. (1999). Informe sobre el estudio de daños que presenta el edificio de la Iglesia parroquial de San Nicolás, sita en el Grao de Gandía, Valencia. Madrid: Intemac.

(6) Lafuente, I. (2001). Proyecto Básico y de Edificación: Restauración de estructura y obras de conservación. Valencia: Colegio Territorial de Arquitectos de Valencia. Visado 16/11/2011

(7) Lafuente, I. (2003). Proyecto Básico y de Edificación: Restauración de estructura y obras de conservación. Segunda Fase. Colegio Territorial de Arquitectos de Valencia. Visado 23/o6/2003

(8) Lafuente, I. (2004). Proyecto Básico y de Edificación: Restauración de estructura y obras de conservación. Tercera Fase. Valencia: Colegio Territorial de Arquitectos de Valencia. Visado 12/11/2004

(9) Sala, R. (2012). Sant Nicolau del Grao de Gandía. 50 Aniversari. Madrid: Instituto Eduardo Torroja.

(10) Krivoshapko, S.N., Bock Hyeng, C.A., Mamieva,I.A.(2014). Chronology of Erection of the Earliest reinforced concrete shells. International. Journal of Research and Reviews in Applied Sciences, 18(2):95-108.

(11) Torroja, E. (1958). The Structures of Eduardo Torroja (1 ${ }^{\mathrm{a}}$ edición). New York: F.W. Dodge Corporation. Edición en español: (1999). Las Estructuras de Eduardo Torroja ( $2^{\text {a }}$ edición). Madrid: Centro de Publicaciones, Ministerio de Fomento.

(12) Chías, P., Abad, T. (2005). Eduardo Torroja. Obras y Proyectos. Madrid: Instituto de Ciencias de la Construcción Eduardo Torroja - CSIC.

(13) Arredondo, F., Benito, C., Echegaray, G., Nadal, J., Páez, A. y del Pozo, F. (1977). La Obra de Eduardo Torroja. Madrid: Instituto de España.

(14) Miñana, J. (1962). El Grao de Gandía y su nueva Iglesia de San Nicolás. Memoria histórico-descriptiva. Valencia: Publicidad Denis.

(15) Echegaray, G. (1959). Proyecto de Iglesia Parroquial y Casa Abadía en el Grao de Gandía. Madrid.

(16) Nadal, J. (1960). Proyecto de estructura para una Iglesia Parroquial. Madrid.

(17) Echegaray, G. (1964). Moderna iglesia: Grao de Gandía, Valencia. Informes de la construcción, 16(157): 5-17.

(18) Cassinello, F. (1974). Construcción. Hormigonería. Madrid: Ed. Rueda.

(19) Cassinello, F. (1961). Estructuras plegadas. Informes de la Construcción, 13(135).

(20) Faber, C. (1970). Las estructuras de Felix Candela. México, España, Argentina, Chile: Compañía editorial continental.

(21) García, R. (2013). Dos décadas de estructuras plegadas de hormigón. Inicio y ocaso de un movimiento. Informes de la Construcción, 65(529): 27-39, doi: http://dx.doi.org/10.3989/ic.11.083.

(22) Azpiazu, J.R. (1971). Experiencias adquiridas a través del proyecto arquitectónico, dirección de obra y construcción de cubiertas laminares. Informes de la Construcción, 24(233): 41-50, doi: http://dx.doi.org/10.3989/ic.1971.v24. i233.3337.

(23) Barredo, R. (1955). Pretensado sistema "Barredo". Informes de la Construcción, 7(70). 
(24) Torroja, E. (1962). Iglesia en Gandía. Informes de la Construcción, 14(137): 48-76.

(25) ASTM International. (2009). ASTM C876-09 Standard Test Method for Corrosion Potentials of Uncoated Reinforcing Steel in Concrete.

(26) AENOR-CEN. (1984). UNE-EN 83302:1984 Ensayos de hormigón. Extracción y conservación de probetas testigo. Asociación Española de Normalización (AENOR).

(27) AENOR-CEN. (1984). UNE-EN 83303:1984 Ensayos de hormigón. Refrentado de probetas con mortero de azufre. Asociación Española de Normalización (AENOR).

(28) AENOR-CEN. (1984). UNE-EN 83304:1984 Ensayos de hormigón. Rotura por compresión. Asociación Española de Normalización (AENOR).

(29) Torroja, E., et al. (1961). Instrucción H.A. 61 especial para estructuras de hormigón armado. Madrid: Instituto Eduardo Torroja de la Construcción y del Cemento.

(30) ASTM International. (2013). ASTM C642-13 Standard Test Method for Density, Absorption, and Voids in Hardened Concrete.

(31) AENOR-CEN. (1994). UNE 112011:1994 Corrosión en armaduras. Determinación de la profundidad de carbonatación en hormigones endurecidos y puestos en servicio. Asociación Española de Normalización (AENOR).

(32) AENOR-CEN. (1994). UNE 112010:1994 Corrosión en armaduras. Determinación de cloruros en hormigones endurecidos y puestos en servicio. Asociación Española de Normalización (AENOR).

(33) AENOR-CEN (1990). UNE 83309:199o Ensayos de hormigón. Determinación de la profundidad de penetración de agua bajo presión. Asociación Española de Normalización (AENOR).

(34) AENOR-CEN (1985). UNE 83306:1985 Ensayos de hormigón. Rotura por tracción indirecta. (Ensayo brasileño). Asociación Española de Normalización (AENOR).

(35) AENOR-CEN (2002). UNE-EN 12004/A1:2002 Adhesivos para baldosas cerámicas. Definiciones y especificaciones. Asociación Española de Normalización (AENOR). 